\title{
An Analysis of the Allocation Scheme for Taxi Resources Using GPS Data
}

\author{
Wei Yang ${ }^{1, a}$, Kun Hou ${ }^{2, b^{*}}$, Yunsheng Zhao ${ }^{3, \mathrm{c}}$, Tian Zhang ${ }^{1, \mathrm{~d}}$ and Zichun Luo ${ }^{2, \mathrm{e}}$ \\ ${ }^{1}$ College of Computer Science and Technology, Changchun Normal University, Changchun \\ 130032, China \\ ${ }^{2}$ School of Information Science and Technology, Northeast Normal University, Changchun 130117, \\ China \\ ${ }^{3}$ School of Geographical Sciences, Northeast Normal University, Changchun 130024, China \\ aviviyw@163.com, bhouk431@nenu.edu.cn, 'zhaoys975@nenu.edu.cn, \\ dzhangtian@cncnc.edu.cn, ${ }^{\mathrm{I}}$ luozc474@nenu.edu.cn \\ *The Corresponding author
}

Keywords: Matching degree of supply and demand for taxi resources; Satisfaction index objective function; Subsidy ratio; Optimization model; GPS

\begin{abstract}
Taxi is one of the important means of transportation for citizens. "Difficulty to take a taxi" is a hot social issue. With the arrival of "Internet+" times, a number of companies have established the taxi service platforms based on the Internet, which have realized the information exchange between the passengers and the taxi drivers, and introduced a variety of taxi subsidy schemes. In order to determine whether the subsidy schemes are helpful for alleviating the problem of "difficulty to take a taxi", taking Beijing as an example, this paper establishes an evaluation system and a passenger satisfaction optimization model based on the taxi density/passenger demand density for the companies' taxi subsidy schemes. Finally, this paper proposes a new subsidy scheme and verifies its rationality and effectiveness.
\end{abstract}

\section{Introduction}

As an important means of transportation, the taxis provide convenient, comfortable and direct transportation services for passengers [1]. There are a huge number of taxis in China, while most urban taxi operations use traditional random mode [1-3]. A lot of roads and energy are wasted [4-6]. With the development and application of third party taxi apps [7-9], taxi operations have gradually formed a market-oriented taxi mode in recent years, which have alleviated the problem of "difficulty to take a taxi". However, the subsidy schemes for taxi apps have problems, which causes serious mismatch between taxi resources and passenger demands. "Difficulty to take a taxi" still exists.

\section{Matching Degree of Supply and Demand for Taxi Resources}

This paper divides Beijing into four regions: A, B, C and D, which are the intersection of the West Second Ring Road and the North Second Ring Road, the Airport Expressway area, and the South Second Ring Road area, as shown in Fig. 1.

Matching degree of supply and demand for taxi resources, MD can be defined as,

$$
\mathrm{MD}=\mathrm{TD} / \mathrm{PD} \times 100 \% \text {. }
$$

where TD denotes taxi density (the number of taxis per unit area) and PD denotes passenger demand density (the number of passengers per unit area).

According to the statistic data of taxis in the Beijing Statistical Yearbook, the "matching degree of supply and demand for taxi resources" in the four regions of Beijing is shown in Fig. 2. The "matching degree of supply and demand for taxi resources" of B region in one day is shown in Fig. 3. 


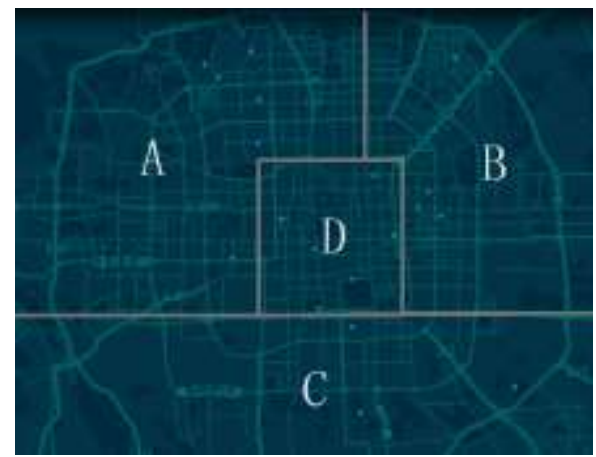

Figure 1. Matching degree of supply and demand for taxi resources

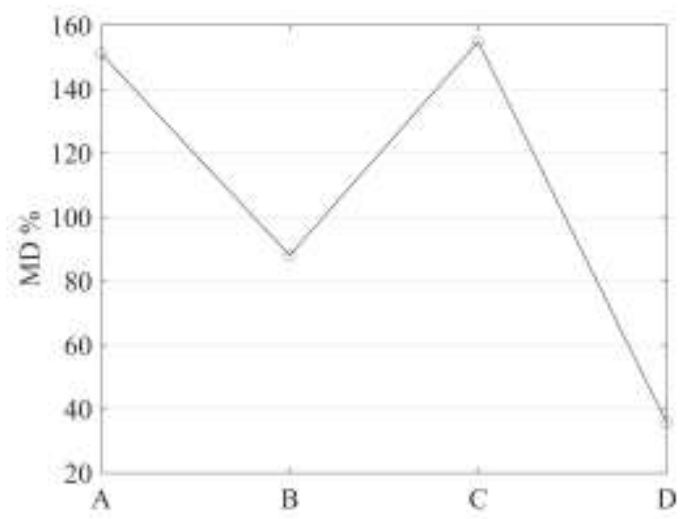

Figure 2. The "matching degree of supply and demand for taxi resources" in the four regions of Beijing

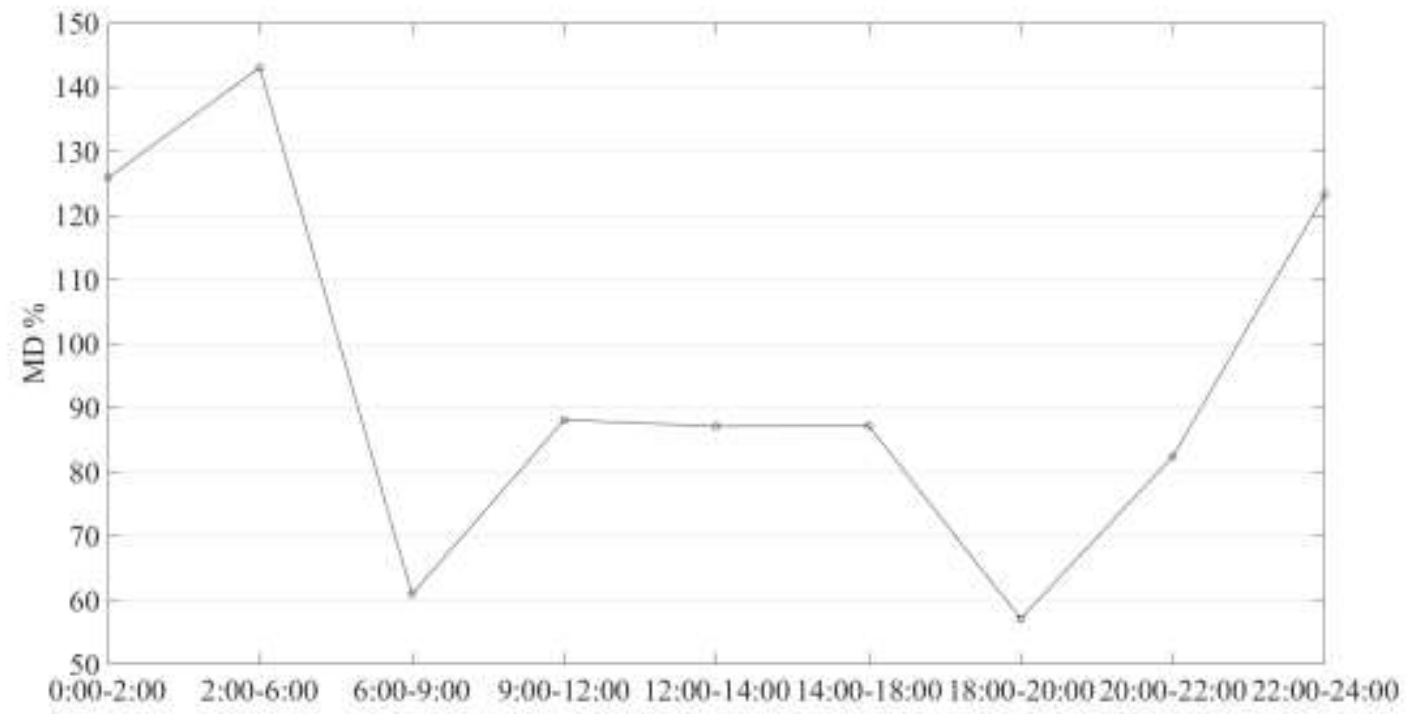

Figure 3. The "matching degree of supply and demand for taxi resources" of B region in one day

It can be seen from Fig. 2 that MD of the B region is the best, followed by the A region and the $\mathrm{C}$ region, and the $\mathrm{MD}$ of the $\mathrm{D}$ region is the worst.

It can be seen from Fig. 3 that in the morning and evening peak hours, the "MD is the worst. During off-peak hours, the MD is roughly the same, of which 9:00-12:00 MD is the best. 


\section{The Impact of the Taxi Subsidy Schemes on MD}

Take the subsidy schemes of Didi taxi app and Fast taxi app as the examples, the drivers are not subsidized during normal times. In peak hours, the drivers' subsidy is 0.2 times the usual cost. The four regions are subdivided into $10 \times 10$ rectangular regions and the number is 100 , and the MD in each rectangular region is calculated to obtain a $10 \times 10 \mathrm{MD}$ matrix. The MD matrix before and after the implementation of the subsidy scheme are shown in Fig. 4 and Fig. 5.

$\left(\begin{array}{llllllllll}513.3621 \% & 1967.8879 \% & 5475.8621 \% & 547.5862 \% & 7015.9483 \% & 855.6034 \% & 684.4828 \% & 684.4828 \% & 15.2786 \% & 34.2241 \% \\ 71.3003 \% & 598.9224 \% & 2139.0086 \% & 867.8264 \% & 1796.7672 \% & 898.3836 \% & 556.1422 \% & 105.6922 \% & 28.5201 \% & 12.2229 \% \\ 1967.8879 \% & 205.3448 \% & 110.8395 \% & 307.6098 \% & 276.4257 \% & 196.9878 \% & 4705.8190 \% & 199.6408 \% & 58.0588 \% & 171.1207 \% \\ 1026.7241 \% & 80.5274 \% & 556.1422 \% & 115.0847 \% & 73.6469 \% & 269.3566 \% & 121.9235 \% & 1454.5259 \% & 770.0431 \% & 598.9224 \% \\ 342.2414 \% & 151.3760 \% & 51.6927 \% & 62.0614 \% & 67.3262 \% & 122.5594 \% & 49.6556 \% & 202.2335 \% & 941.1638 \% & 128.3405 \% \\ 85.5603 \% & 85.5603 \% & 1796.7672 \% & 195.5665 \% & 143.6192 \% & 360.7409 \% & 453.4698 \% & 69.5178 \% & 108.3764 \% & 4.5632 \% \\ 34.2241 \% & 31.5222 \% & 20.5345 \% & 22.0801 \% & 17.6663 \% & 11.3850 \% & 45.2967 \% & 260.4010 \% & 19.0134 \% & 1.4260 \% \\ 684.4828 \% & 342.2414 \% & 85.5603 \% & 342.2414 \% & 140.9229 \% & 54.4475 \% & 7.3337 \% & 171.1207 \% & 7443.7500 \% & 427.8017 \% \\ 6.1115 \% & 85.5603 \% & 4.3877 \% & 85.5603 \% & 70.0039 \% & 85.5603 \% & 2.6738 \% & 705.8728 \% & 513.3621 \% & 171.1207 \% \\ 2.4446 \% & 8.5560 \% & 85.5603 \% & 73.8930 \% & 85.5603 \% & 85.5603 \% & 770.0431 \% & 171.1207 \% & 85.5603 \% & 256.6810 \%\end{array}\right)$

Figure 4. MD matrix before the implementation of the subsidy scheme

$\left(\begin{array}{llllllllll}22.1927 \% & 798.9379 \% & 2529.9700 \% & 4.4385 \% & 798.9379 \% & 22.1927 \% & 22.1927 \% & 22.1927 \% & 0.7926 \% & 4.4385 \% \\ 3.6988 \% & 22.1927 \% & 654.6852 \% & 350.3279 \% & 754.5524 \% & 166.4454 \% & 5.5482 \% & 1.3055 \% & 1.4795 \% & 0.6341 \% \\ 22.1927 \% & 4.4385 \% & 82.2139 \% & 306.4709 \% & 481.4113 \% & 362.3090 \% & 6657.8157 \% & 239.1882 \% & 250.4607 \% & 1731.0321 \% \\ 22.1927 \% & 154.0436 \% & 1120.7323 \% & 103.3055 \% & 75.5676 \% & 358.3713 \% & 178.3740 \% & 7234.8264 \% & 2308.0428 \% & 22.1927 \% \\ 22.1927 \% & 1.7071 \% & 8.5534 \% & 62.9836 \% & 22.1927 \% & 93.7692 \% & 72.1263 \% & 272.3652 \% & 998.6723 \% & 5.5482 \% \\ 22.1927 \% & 2.7741 \% & 22.1927 \% & 412.1505 \% & 414.5283 \% & 584.2083 \% & 356.1931 \% & 1.3870 \% & 1.4795 \% & 0.2959 \% \\ 4.4385 \% & 45.5535 \% & 0.8877 \% & 0.3579 \% & 46.8114 \% & 17.6289 \% & 43.5151 \% & 102.2795 \% & 0.4110 \% & 0.0925 \% \\ 22.1927 \% & 22.1927 \% & 22.1927 \% & 22.1927 \% & 1.3055 \% & 1.0088 \% & 0.6341 \% & 324.7145 \% & 22.1927 \% & 22.1927 \% \\ 1.5852 \% & 22.1927 \% & 0.5690 \% & 5.5482 \% & 238.0673 \% & 22.1927 \% & 0.6935 \% & 493.7880 \% & 5659.1433 \% & 22.1927 \% \\ 0.6341 \% & 2.2193 \% & 22.1927 \% & 1.0088 \% & 5.5482 \% & 22.1927 \% & 22.1927 \% & 22.1927 \% & 22.1927 \% & 22.1927 \%\end{array}\right)$

Figure 5. MD matrix after the implementation of the subsidy scheme

It can be seen from Fig. 4 and Fig. 5 that the area where the MD value tends to 1 has an expanding trend after the implementation of the subsidy scheme, which indicates that the implementation of the subsidy scheme is helpful for alleviating the problem of "difficulty to take a taxi". However, the difference in MD value between 100 rectangular region is still very large and the "matching degree of supply and demand for taxi resources" is still very low. The subsidy scheme has not fundamentally alleviated the problem of "difficulty to take a taxi".

\section{Passenger Satisfaction Subsidy Scheme}

In this paper, passenger satisfaction is used as the dependent variable, and MD is used as the independent variable. A mathematical model of passenger satisfaction with optimized functions can be denoted by,

$$
\begin{aligned}
& \text { Maximize } V(x) \\
& V(x)=v_{1} \times x_{1}+v_{2} \times x_{2}+\ldots+v_{n} \times x_{n} \\
& \text { Subject to } a_{1} \leq x_{1} \leq b_{1}, a_{2} \leq x_{2} \leq b_{2}, \ldots, a_{n} \leq x_{n} \leq b_{n}, 1 \leq i \leq 100
\end{aligned}
$$


where $\mathrm{V}(\mathrm{x})$ is the weighted average passenger satisfaction, $\mathrm{v}_{\mathrm{i}}$ is the weighted average passenger satisfaction in the $\mathrm{i}$-th rectangular region, and $\mathrm{x}_{\mathrm{i}}$ is the fluctuation range of the MD value in the $\mathrm{i}$-th rectangular region. The remaining fixed constraints include: the number of taxis, the total cost of subsidies and the number of passengers.

Ranking according to the ratio of the MD values in 100 region,

According to the ratio of the MD values in 100 regions, the satisfactory index objective function (Sigmoid function [10]) model is defined as follows,

$\mathrm{Y}=1 / 2+1 / \pi \times \arctan 4(\mathrm{x}-1)$.

The subsidy ratio (SR) objective function model can be denoted by,

$\mathrm{Y}=1 / 2-1 / \pi \times \arctan 4(\mathrm{x}-1)$.

With the introduction of the satisfaction index objective function, the passenger satisfaction index (PSI) increases with the increase of the MD value, and in the vicinity of the MD value of 1 , the satisfaction index rises the fastest. When the MD value tends to zero, the passenger satisfaction index will also be forced to zero. The variation law of Sigmoid function image can reflect the function image law of passenger satisfaction index and MD value. As shown in Fig. 6.

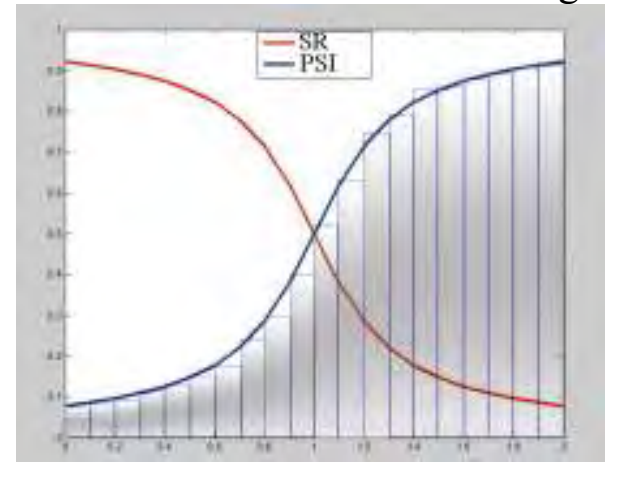

Figure 6. SR and PSI

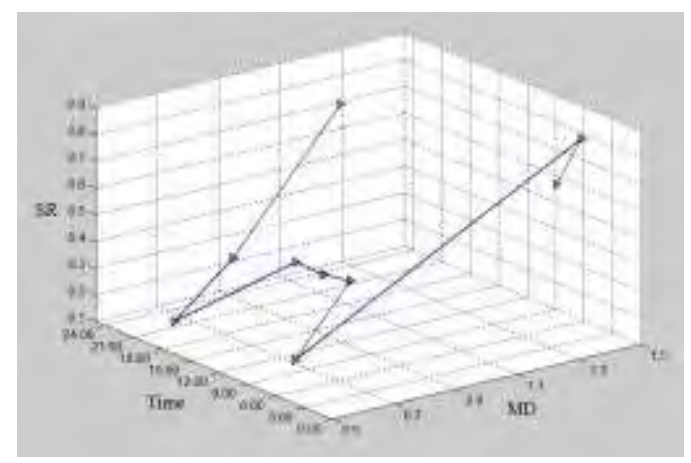

Figure 7. Three-dimensional map of MD and SR in different times

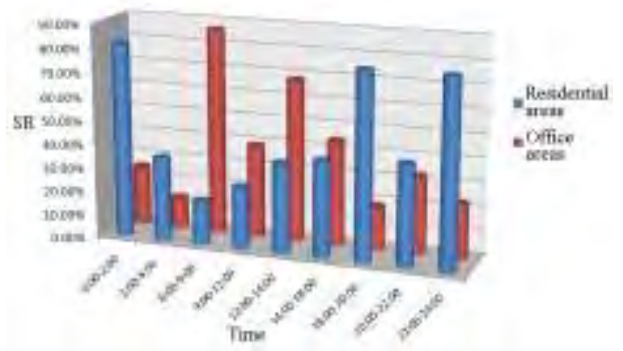

Figure 8 . Three-dimensional map of the MD and SR in different regions 
It can be seen from Fig. 6 that PSI is positively correlated with MD, and the SR is negatively correlated with MD. Therefore, in our subsidy scheme, we need to increase the SR in regions with small $\mathrm{MD}$; and reduce the SR in regions with large MD.

Fig. 7 is a three-dimensional map of MD and SR in different times (including peak times and normal times). Fig. 8 is a three-dimensional map of MD and SR in different regions (including residential areas and office areas).

Fig. 7 and Fig. 8 generated from the sample GPS data reflect the relationship between SR and MD, which will be an important basis for establishing models and verification models. This paper will make reasonable assumptions and formalize the problem into the previous optimization problem. The specific modeling scheme is as follows:

1. The amount of funds that each company can invest in is limited, assuming W (Worth) represents the total amount of money a company can invest in an administrative area each day. Think of it as a fixed value in the model of this paper. In addition, the total demand is different at different times, for which time the time of day is divided into several time segments, and $\mathrm{T}$ represents the length of the time segment. In this paper, $\mathrm{T}$ (Time) takes $10 \mathrm{~min}$.

2. Based on past GPS data, the trend of future demand over time can be predicted. Figure 9 gives the forecast results.

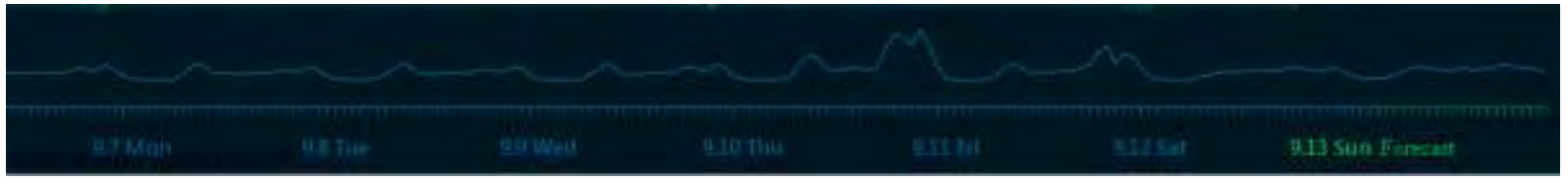

Figure 9. The trend of future demand

3. After the definite integral value of the prediction function is obtained, the ratio Ratio Predict $\left(\mathrm{R}_{\mathrm{P}}\right)$ of the total integral value of the different time segments is obtained. Thereby, the change function step function $\left(\mathrm{F}_{\mathrm{R}}\right)$ of the ratio with time can be obtained. If let WT indicate the total amount of money allocated to different time periods, then $\mathrm{W}_{\mathrm{T}}=\mathrm{W} \times \mathrm{F}_{\mathrm{R}}$.

4. The nature of each time period is discussed below. Since the average time of each time a taxi is completed, that is, the average time of each passenger's travel time is greater than 10 minutes, it is assumed that the number of individual taxis in a single time period is 1 , and the direction of travel of the taxi in each time period. Can be approximated as fixed.

5. The administrative area is divided into sub-areas according to the nature of the service. Firstly, the density of taxis in different sub-areas in a single time period is obtained by GPS data, and then the demand density of taxis in different sub-areas in a single time period is estimated by monitoring data of taxis, number of software orders and location distribution. Finally, the ratio of the taxi density to the demand density in different sub-areas in a single time period is obtained, which is recorded as $R_{D / N}$ (Ratio Density/Need).

6. First, calculate the sum of the ratios of the administrative regions, and then calculate the ratio of the ratio $R_{D / N}$ of the taxi density to the demand density in different sub-regions in a single time period to the percentage ratio $\mathrm{P}$ (percent). In order to facilitate the calculation of $\mathrm{P} \times \mathrm{W}_{\mathrm{T}}, \mathrm{P}$ is arranged from small to large. Use $M_{P}$ to indicate the amount of subsidy allocated to different sub-areas in each single transaction. The larger the $R_{D / N}$, the easier it is to take a taxi, so the subsidy should be less, that is, $R_{D / N}$ is inversely proportional to $M_{P}$. The $R_{D / N}$ is ranked from high to low, and corresponds to the sorted $\mathrm{P} \times \mathrm{W}_{\mathrm{T}}$, and finally the subsidy amount $\mathrm{M}_{\mathrm{P}}$ assigned to different sub-areas in each single transaction is obtained. Therefore, we get a newly reasonable subsidy scheme.

\section{Conclusion}

This paper fully considers the impact of waiting time on passenger psychology to establish a dynamic model of the relationship between subsidy ratio and satisfaction index. The analysis of "matching degree of supply and demand for taxi resources" fundamentally solves the problem of "difficulty to take a taxi". 
However, in the study of passenger satisfaction, the model does not analyze the impact of taxi app on passenger satisfaction and "difficulty to take a taxi". The division of the $10 \times 10$ grid area adopted by Beijing in the analysis of passenger demand density is still not fine enough.

Although the model established in this paper is based on the operation status of Beijing taxis, it can be applied to cities with different development levels. In further research in the future, it can be more subdivided when dividing the sub-region grid, for example, using a granularity of $100 \times 100$. In addition, when formulating the subsidy scheme, it can be flexibly determined according to the city's income level the dynamic model of the relationship between the subsidy ratio and the satisfaction index.

\section{Acknowledgements}

This work was supported in part by the Jilin Provincial Education Department's "Thirteenth FiveYear" scientific and technological research planning project under Grant JJKH20190500KJ, and in part by the Natural Science Foundation of Changchun Normal University.

\section{References}

[1] C. Kamga, M.A. Yazici and A. Singhal: Transportation Planning And Technology, Vol.38 (2015) No.6, p.601.

[2] C. Yang and E.J. Gonzales: Transportation Research Record, Vol.2429 (2014) No.2429, p.110.

[3] L. Moreira-Matias, J. Gama, M. Ferreira, J. Mendes-Moreira and L. Damas: IEEE Transactions on Intelligent Transportation Systems, Vol.14 (2013) No.3, p.1393.

[4] X. Hu, S. An and J. Wang: Journal Of Advanced Transportation, Vol.2018 (2018) No.1, p.1.

[5] J.-P. Jokinen: Journal Of Transport Economics And Policy, Vol.50 (2016) No.1, p.39.

[6] R. Su, Z. Fang, N. Luo and J. Zhu: Sustainability, Vol.10 (2018) No.4, p.1256.

[7] J. Xu, R. Rahmatizadeh, L. Boloni and D. Turgut: IEEE Transactions on Intelligent Transportation Systems, Vol.19 (2018) No.8, p.2572.

[8] X. Chen, C. Chen, L. Ni and L. Li: Physica A Statistical Mechanics And Its Applications, Vol.508 (2018) No.C, p.84.

[9] J.Y. Chen: New Media \& Society, Vol.20 (2018) No.8, p.2691.

[10]D. Feingold, S.R. Steele, S. Lee, A. Kaiser, R. Boushey, W.D. Buie and J.F. Rafferty: Diseases Of the Colon \& Rectum, Vol.57 (2014) No.3, p.284. 